\title{
REINTERPRETING POLYMER UNFOLDING EFFECT INDUCED BY A SPATIALLY CORRELATED NOISE*
}

\author{
M. MAJKA, P.F. Góra \\ The M. Smoluchowski Institute of Physics, Jagiellonian University \\ Reymonta 4, 30-059 Kraków, Poland
}

(Received May 10, 2013)

This paper provides additional insight into the effect of spontaneous unfolding of the model polymeric chain driven by spatially correlated noise, described in M. Majka, P.F. Góra, Phys. Rev. E86, 051122 (2012). We examine the statistical data on the linearized chain substructures to find that the global unfolding effect arises mainly from the cumulation of short, 2-segment-long fragments, scattered along the chain. This supports an alternative view of spatially correlated noise as both the source of disturbance and the conformation preserving factor.

DOI:10.5506/APhysPolB.44.1099

PACS numbers: 05.40.Ca, 36.20.-r, 61.43.Fs, 87.15.A-

\section{Introduction}

Noise induced phenomena e.g. spontaneous ordering induced by white noise [1] or the system synchronization induced by colored noise [2] are of great concern for contemporary research. However, while the white noise and Langevin dynamics are standard tools in complex system simulations [1] and also the time-correlated noise combined with Generalized Langevin Equations becomes increasingly popular in applications [3], little research has been devoted to the spatially correlated noise.

The spatially correlated noise is a disturbance which is random at large length-scale, but ordered at the length-scale of correlation length $\lambda$. This kind of disturbance forms a space pattern which can evolve over time in a completely random or temporally correlated manner, tough preserving a certain ordering below the length $\lambda$. Such noise is designed to resemble

* Presented at the XXV Marian Smoluchowski Symposium on Statistical Physics, "Fluctuation Relations in Nonequilibrium Regime", Kraków, Poland, September 10-13, 2012. 
a collectively acting heat-bath affecting a sub-system of interest with possible application to soft matter simulations. Random, yet spatially correlated phenomena are encountered especially in the research on glass-forming [4-6], active particles swimming [7, 8] and sedimentation in crowded solutions $[9,10]$.

Our main results regarding the influence of spatially correlated noise on the model two dimensional polymeric chain has been already published in [11] and [12]. The paper [11] is a primary reading which provides the most complete introduction to the topic as well as full support for our findings, and we shall relate to this article repeatedly in the present work. Thus, it is preferable that the Reader will familiarize himself with [11] before proceeding. Nevertheless, we should present here a brief summarization of the most important notions from [11] to facilitate the reading.

We have examined a two dimensional polymer chain model based on the bead-spring approach with the nearest neighbor, angular and excluded volume interactions. The position of $i$ th bead has been simulated according to the equations of motion

$$
\left\{\begin{aligned}
m \ddot{x}_{i}+\gamma \dot{x}+\partial_{x_{i}} U & =\xi_{x}\left(\vec{r}_{i}, t\right), \\
m \ddot{y}_{i}+\gamma \dot{y}+\partial_{y_{i}} U & =\xi_{y}\left(\vec{r}_{i}, t\right) .
\end{aligned}\right.
$$

Here $m$ is a bead mass, $\gamma$ is a friction constant and $U$ is total potential energy (to be specified in Section 2). While we have kept inertial terms in (1) for the sake of numerical accuracy, the constants were chosen so $\gamma / m=20$, which was enough to over-damp the system. $\vec{\xi}(\vec{r}, t)$ denotes the spatially correlated Gaussian noise, whose correlation function reads

$$
\begin{aligned}
& \left\langle\xi_{x}\left(\overrightarrow{r_{i}}, t_{2}\right) \xi_{x}\left(\overrightarrow{r_{j}}, t_{1}\right)\right\rangle=\left\langle\xi_{y}\left(\overrightarrow{r_{i}}, t_{2}\right) \xi_{y}\left(\overrightarrow{r_{j}}, t_{1}\right)\right\rangle=\sigma \frac{\gamma}{m} e^{-\frac{\left|\vec{r}_{i}-\vec{r}_{j}\right|}{\lambda}} \delta\left(t_{2}-t_{1}\right), \\
& \left\langle\xi_{x}\left(\overrightarrow{r_{i}}, t_{2}\right) \xi_{y}\left(\overrightarrow{r_{j}}, t_{1}\right)\right\rangle=0 .
\end{aligned}
$$

Here, $\sigma$ is temperature and $\lambda$ is a correlation length.

We have found that the presence of spatial correlations in the noise results in several measurable effects, which are: chain stiffening (discussed comprehensively in [12]), the synchronization of beads motion, increased time correlation of segments lengths and angles between segments, and the spontaneous unfolding of the chain [11].

The effect of spontaneous unfolding is of special interest in this paper, and we would like to discuss it now in a greater detail. In [11], we have presented the distribution of angles between neighboring polymer segments (defined by equation (10), Section 2) for a range of temperatures and correlation lengths. For sufficiently high temperatures, these data proved to be single peaked distributions, symmetric around their mean values. The peak 
in probability is associated with neighboring segments lying exactly in line, yet these distributions are broad and, especially for the uncorrelated noise, the raise in $\sigma$ increases the variance of distribution. Having introduced spatial correlations into noise, the distributions become remarkably narrower and, for constant temperature, the longer correlation length is, the lower is the variance. In the range of correlation lengths which we have tested (approximately from 1 to 7 segment equilibrium lengths), this fall of variance seems to asymptotically approach certain minimal, yet non-zero, value. Additionally, for constant and non-zero correlation length, the temperature dependent growth of variance is significantly mitigated in comparison to the uncorrelated case.

From the preceding description, one can conclude that neighboring segments tends to linearize, yet the distribution of angles gives no information what structures prevail in the chain geometry to give rise to this effect, and, especially, how long those linearized fragments are. We will elaborate on this in Section 3. In Section 4, we will outline a new interpretation of the unfolding effect, alternative to one given in [11]. However, first, we would like to present our polymer model in Section 2.

\section{Polymer chain model}

Our polymer chain model consists of $N$ interacting material points (beads) for which we have applied the Langevin dynamics. The beads interact via three kinds of potential that form a bead-spring model enriched with angular and excluded volume interactions. The total potential energy of the system reads

$$
U=U_{R}+U_{\psi}+U_{\mathrm{LJ}} .
$$

$U_{R}$ is the nearest neighbor interaction, which resembles bonds, and it is defined by

$$
U_{R}=\sum_{i=1}^{N-1} \frac{1}{2} k_{1}\left(\left|\vec{r}_{i+1}-\vec{r}_{i}\right|-d_{0}\right)^{2} .
$$

Here, $\vec{r}_{i}$ is a position of the $i$ th bead and $d_{0}$ is an equilibrium segment length. The angular interaction $U_{\psi}$ is modeled with the second nearest neighbor interaction, which is

$$
U_{\psi}=\sum_{i=1}^{N-2} \frac{1}{2} k_{2}\left(\left|\vec{r}_{i+2}-\vec{r}_{i}\right|-l_{0}\right)^{2} .
$$

In this case, $l_{0}$ defines the preferable distance between $i$ th and $i+2$ nd bead, and to ensure a saw-like chain conformation, it should be satisfied that [11]

$$
l_{0}<2 d_{0} .
$$


Finally, we introduce the global Lennard-Jones interaction $U_{\mathrm{LJ}}$

$$
U_{\mathrm{LJ}}=\sum_{i, j}^{N} \epsilon\left(\frac{\sigma_{\mathrm{LJ}}^{12}}{\left|\vec{r}_{i}-\vec{r}_{j}\right|^{12}}-\frac{\sigma_{\mathrm{LJ}}^{6}}{\left|\vec{r}_{i}-\vec{r}_{j}\right|^{6}}\right) .
$$

This interaction brings in the effect of excluded volume thanks to its repulsive core (its diameter is proportional to $\sigma_{\mathrm{LJ}}$ ) and provides the attraction whenever the distant parts of chain close up. Parameters $k_{1}, k_{2}$ and $\epsilon$ are scaling constants and their exact values used in simulations are gathered in Table I.

TABLE I

The parameters of the system chosen for simulations. These parameters provided the saw-like chain conformation, presence of repulsive core for each bead and the over-damping of beads' motion.

\begin{tabular}{ccccccccc}
\hline \hline$N$ & $k_{1}$ & $d_{0}$ & $k_{2}$ & $l_{0}$ & $\epsilon$ & $\sigma_{\mathrm{LJ}}$ & $\gamma$ & $m$ \\
\hline 128 & 7 & 7 & 2 & 11 & 1 & 3 & 20 & 1
\end{tabular}

For the detailed discussion of the minimal energy structures, a single bead energy landscape and a chain geometry dynamics, please see [11], but here we would like to outline only the most important facts. A single bead moves in a potential well determined by the positions of its neighboring beads. For parameters chosen according to Table I, this well has most often a double minimum, so there are two possible positions of a bead relative to the rest of the chain that minimize the bead's energy. Both, the depth of each minimum and their exact positions change as the local geometry evolves, yet the double-well landscape is predominant and fairly invulnerable to changing angles between segments. However, this double-well structure is extremely sensitive to the increase in distance between the nearest neighbors of the bead. Such stretching causes the two minima to merge rapidly into one, which is positioned in line with the nearest neighbors. This is seen as a local frustration of the chain, because the minimal energy structures never tend to linearize.

In [11], the conformational dynamics of chain is examined primarily with two parameters, namely the length of the segment

$$
d_{j}(t)=\left|\vec{r}_{j}(t)-\vec{r}_{j-1}(t)\right|
$$

and the angle between neighboring segments, which has been obtained from positions $\vec{r}_{j}$ of the three following beads

$$
\psi_{j}=\angle\left(\vec{r}_{j-1}, \vec{r}_{j}, \vec{r}_{j+1}\right) \text {. }
$$


This angle is directed, varies from 0 to $360^{\circ}$ and it is always measured with respect to certain initial numeration of beads. It is also scaled, so $\psi_{j}=180^{\circ}$ indicates three beads being exactly in-line. We have found that for parameters from Table I and low $(3<\sigma<12)$ uncorrelated noise, the system prefers the saw-like conformation, with $d_{j} \approx d_{0}$. For such $\sigma$, the distribution of angles $\psi_{j}$ is symmetric, double peaked, with one peak approximately at $110^{\circ}$ and the other at $250^{\circ}$. This is consistent with the double-well picture, described in the previous paragraph. With the rise in temperature above $\sigma=13$, the two peaks melt down and the third peak appears exactly at $\psi_{j}=180^{\circ}$. This process is gradually retarded with the rise in noise's spatial correlation length, e.g. for $\lambda=50$ the single peaked distribution appears as late as for $\sigma>25$. From now on, the distribution of $\psi_{j}$ evolves as described in the introductory section, giving rise to the effect of unfolding.

In general, the prevalence of mono-peaked $\psi_{j}$ distributions can be explained by the presence of repulsive Lennard-Jones cores. The rise in temperature increases the mobility of beads, but such cores prevent beads from closing up. Effectively, average distances between beads tend to grow and many sites along the chain suffer frustration, which means that these sites are govern by the single-minimum energy landscape. In other words, the random disturbance can much easier stretch the system than squeeze it. While such mechanism could be accepted for the uncorrelated noise, which provides relative forcing at all length-scales, it is not clear why it should be also valid for the spatially correlated noise, which introduces low relative forcing below correlation length. Namely, the ability of noise to compress or extend the system at the length-scale of $\lambda$ is remarkably reduced, and, in fact, in [11], it has been shown that spatial correlations mitigate the growth in average distances. Therefore, for $\lambda \neq 0$ the chain should be less affected by the noise, but $\psi_{j}$ indicates the opposite. In order to explain this contradiction, we would like to provide another data, regarding the length of linearized fragments and their distribution along the chain.

\section{Chain substructures}

Having determined $\psi_{j}$ angles in one particular moment, it is also possible to count the lengths of linearized fragments of the chain. Two neighboring segments are treated as linearized if the angle between them satisfies $\psi_{j} \in$ $\left[160^{\circ}, 200^{\circ}\right]$. The length of a linearized fragment is defined as the number $n$ of following beads that sequentially fulfill the given criterion. Scanning along the chain, a single segment is assigned to the longest linearized fragment it belongs to. Repeating the scans for different moments leads to the statistics of such fragments' lengths. A single, non-linearized segment is taken as the fragment with $n=1$, so only fragments with $n \geq 2$ are truly indicating linearization. 
We have conducted simulations for $\sigma$ ranging from 1 to 1000 and $\lambda$ from 0 to 50 . In the range $1 \leq \sigma \leq 20$, with the temperature increment equal 1 , we have varied $\lambda$ from 0 to 20 by 5 units. For $25 \leq \sigma \leq 250$, the $\sigma$ increment was 25 and in the interval $300 \leq \sigma \leq 1000$ the increment was equal 100. For these two temperature ranges, the correlation length has been varied by 10 from 0 to 50. For each pair of $\sigma$ and $\lambda$, we have performed 64 runs, from which the statistics has been averaged out. During each run, which lasted $2^{18}$ steps, we have measured statistics every 32 steps. There have been also $100 \times 2^{7}$ initial steps for system thermalization, during which there has been no acquisition of data.

We have collected data regarding the abundance of $n$-segment-long linearized fragments in the form of a histogram. The value $P_{n}$ of the $n$th bin estimates the probability that a single segment belongs to an $n$-segmentlong linearized fragment, with $P_{1}$ occupied by non-linearized segments. For
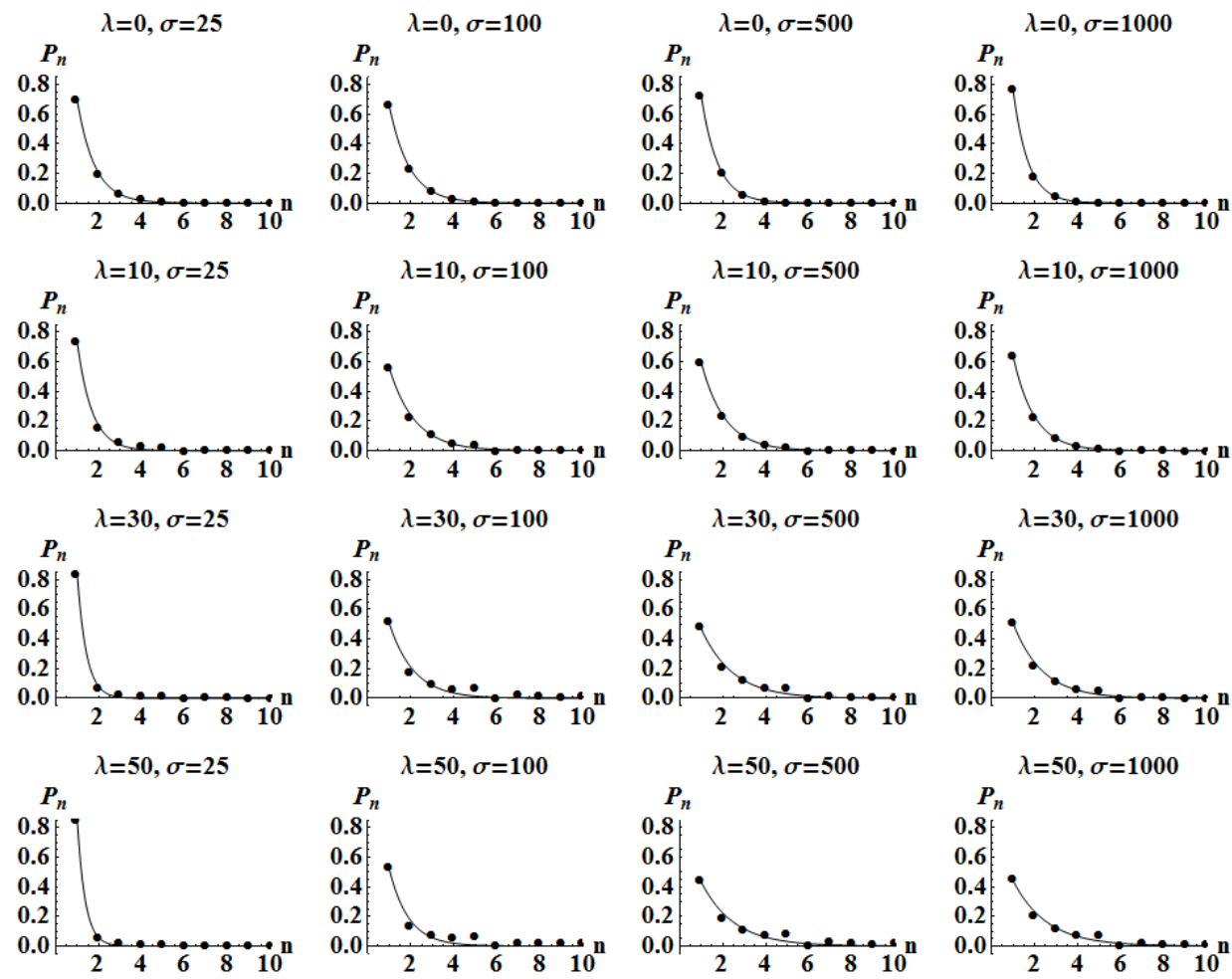

Fig. 1. The comparison of histograms $P_{n}$ for temperatures $25 \leq \sigma \leq 1000$ and correlation lengths $0 \leq \lambda \leq 50$. Data from simulations (black dots) has been fitted with exponential decay function (solid line). With increasing $\sigma$ histograms approach exponential distribution. 
selected temperatures, these data are presented in Fig. 1. Histograms have been fitted with an exponential decay function

$$
p(n)=A \exp \left(-\frac{n-1}{\kappa}\right) .
$$

This model proved to fit the data exceptionally well for $\lambda=0$ and $\lambda=10$ with $\sigma>75$, but for lower temperatures fits became inaccurate. This is also the problem for larger $\lambda$, for which fits seem to gradually improve with the rise in $\sigma$, yet this process is spanned over even larger temperature interval (see Fig. 1). The general trend of $P_{n}$ is to exceed the values predicted with $p(n)$, thus the exponential decay model is a lower boundary approximation at best. Nevertheless, Fig. 1 suggests that exponential distribution is correct at least in the large temperature limit. The retardation of the temperature dependent evolution has been also described in [11], regarding the angles distributions, thus it is no surprise to encounter it in the current dataset.

Despite discussed limitations, the model (11) provides a reasonable approximation for $n=1,2,3$-long fragments, which are the most abundant. Parameter $\kappa$ from equation (11) indicates overall trend in the linearization effect, and its relative error has been lower that $5 \%$ for all fitted curves. In Fig. 2, we have visualized $\kappa$ with respect to the correlation length and temperature. In general, the behavior of $\kappa$ is complicated, yet for $\sigma>300$, it is evidently growing with the rise in $\lambda$ from approximately 0.7 to 1.6. This is clear indication that the participation of short, linearized structures is $\lambda$ dependent and increases with the growing correlation length.

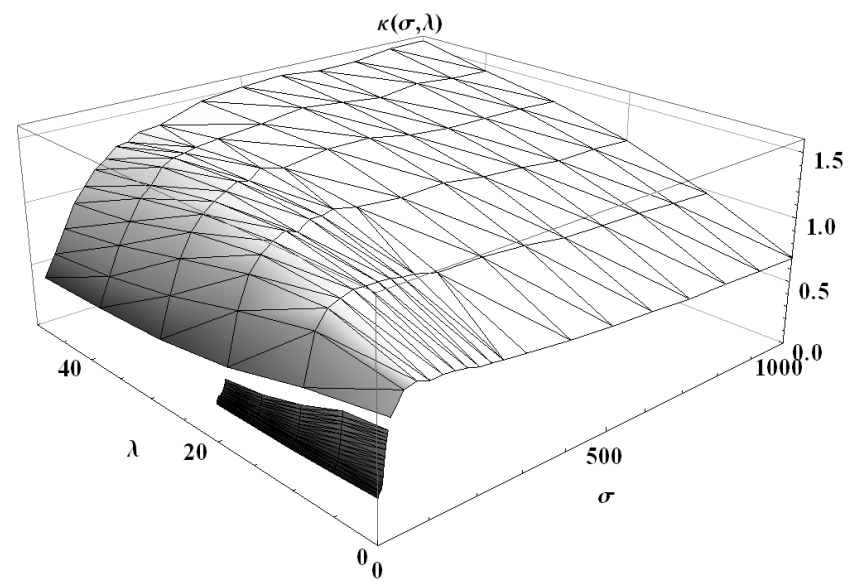

Fig. 2. Decay constant $\kappa$ (see equation (11)) as a function of temperature $\sigma$ and correlation length $\lambda$. For $\sigma>300$, the $\kappa$ is growing with $\lambda$, which reflects the increasing linearization of the chain. 
We have also compared $P_{n}$ for $n=2,3,4$ in relation to $\lambda$ and $\sigma$ (Fig. 3). Typically, for $\sigma>300$ and $\lambda \geq 10, P_{2}$ exceeds $0.16, P_{3}$ is approximately equal to 0.1 and $P_{4}$ reaches 0.06 , which means that 2 to 4 long fragments are dominant structures and usually contain around 30\% of segment population. In general, from $15 \%$ to $45 \%$ of segments form linearized structures, so fragments which are even longer than 4 are also present, yet significantly less abundant (e.g. $P_{6}$ is always smaller than 0.01 ) and they are not vital for the unfolding effect. Additionally, knowing that two linear fragments are separated by at least one non-linearized segment, we can suppose that the linearized fragments are distributed along the chain more or less uniformly. This means that the unfolding effect is a global process occurring parallel at multiple sites and based mainly on the short linear substructures.

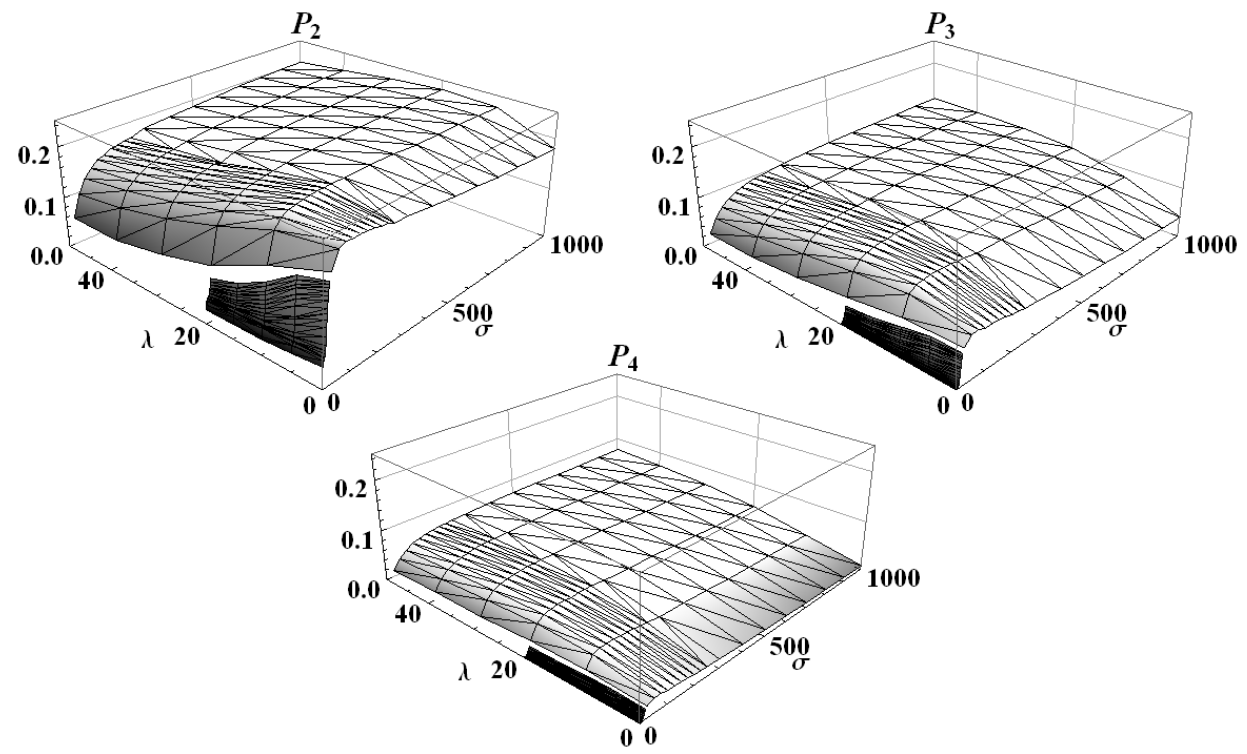

Fig. 3. $P_{n}$ is a probability that a given chain segment belongs to an $n$-long linearized segment. Above, a comparison between $P_{2}, P_{3}$ and $P_{4}$ showing the abundance of these structures and their dependence on temperature $\sigma$ and correlation length $\lambda$.

\section{Reinterpretation of chain unfolding}

Now, we would like to propose a reinterpretation of the chain unfolding process. As it has been stated in Section 2, local linearization indicates the single minimum energy landscape and can be interpreted as a frustration. The spatially correlated noise provides low relative forcing at the distances below $\lambda$, and, indeed, it does not directly affect the low-level structure itself. 
However, the fragments of chain which are larger than $\lambda$ can move in an uncorrelated manner. As a result of this incoherent motion, there appear frustrated sites, and they should occur randomly and uniformly along the chain. The exact positions of these frustrated sites depend only on the random realization of spatial noise pattern at certain time moment. Yet, this pattern evolves rapidly, so a single site that has been once frustrated can found itself in the middle of region which is not affected by the noise, because of spatial correlations. In this case, the lack of relative forcing hinders a local relaxation process, and so frustration is stabilized. In turn, frustrated sites are accumulated along the chain, which is manifested as the unfolding effect. Therefore, the interplay between frustrated sites production and the shape preserving influence of spatially correlated noise could be a main source of the unfolding effect. Such notion is in agreement with our data, both those presented in [11] and those discussed in previous section.

\section{REFERENCES}

[1] F. Sagués, J.M. Sancho, J. García-Ojalvo, Rev. Mod. Phys. 79, 829 (2007).

[2] R. Morgado, M. Cieśla, L. Longa, F.A. Oliveira, Eur. Phys. Lett. 79, 10002 (2007).

[3] S.C. Kou, Ann. Appl. Stat. 2, 501 (2008).

[4] C. Donati et al., Phys. Rev. E60, 3107 (1999).

[5] B. Doliwa, A. Heuer, Phys. Rev. E61, 6898 (2000).

[6] A.C. Mitus, A.Z. Patashinski, A. Patrykiejew, A. Sokolowski, Phys. Rev. B66, 184202 (2002).

[7] P.T. Underhill, J.P. Hernandez-Ortiz, M.D. Graham, Phys. Rev. Lett. 100, 248101 (2008).

[8] P. Romanczuk et al., Eur. Phys. J. Special Topics 202, 1 (2012).

[9] P.N. Segré, E. Herbolzheimer, P.M. Chaikin, Phys. Rev. Lett. 79, 2574 (1997).

[10] E. Guazzelli, Phys. Fluids 13, 1537 (2001).

[11] M. Majka, P.F. Góra, Phys. Rev. E86, 051122 (2012).

[12] M. Majka, P.F. Góra, Acta Phys. Pol. B 43, 1133 (2012). 